\title{
Complete Shrinkage of the Obliterated False Lumen After Open and Endovascular Chronic Aortic Dissection Stanford Type A Repair
}

\author{
George Samanidis, PhD*, Meletios Kanakis, MD, PhD, Constantinos leromonachos, MD, \\ George Stavridis, FECTS
}

Department of Cardiac Surgery, Onassis Cardiac Surgery Center, Athens, Greece

\begin{abstract}
A 48-year-old man was admitted to our hospital with chronic aortic dissection Stanford Type A. His diagnosis was confirmed by chest multi-detector computed tomography (CT). The patient underwent combined (i.e., hybrid) open and endovascular repair (frozen elephant trunk) in a one-stage operation with moderate hypothermic circulatory arrest and antegrade cerebral perfusion. His postoperative course was uneventful, and he was discharged home on postoperative day 9. At 2-year follow-up, chest CT angiography revealed complete shrinkage of the obliterated false lumen in the distal aortic arch and descending thoracic aorta.

Copyright (c) 2017 Science International Corp.
\end{abstract}

\section{Key Words:}

Aortic dissection - Chronic aortic dissection Hypothermic circulatory arrest

We present a case of a 48-year-old man who underwent elective combined (i.e., hybrid) open and endovascular repair of chronic aortic dissection Type A (frozen elephant trunk with endovascular stent) in a one-stage operation with moderate hypothermic circulatory arrest and antegrade cerebral perfusion (ACP). His past medical history included a 7-monthold known aortic dissection Type A, hypertension, and smoking. Preoperative transthoracic echocardiography showed left ventricular ejection fraction of

Published by Science International Corp. ISSN 2325-4637

Fax +1 2037853552

E-Mail: aorta@scienceinternational.org

http://aorta.scienceinternational.org

Accessible online at:

http://aorta.scienceinternational.org
$58 \%$ with dissection of the ascending aorta above the sinotubular junction extending to the aortic arch. Preoperative computed tomography (CT) angiography of the aorta revealed aortic dissection of the ascending aorta extending to the descending thoracic and abdominal aorta (Figures 1IA, 1IB, 1IC, 1ID and Figure 2A). The dimensions of the aortic root (sinus of Valsalva level), ascending aorta, aortic arch, and descending aorta were $40 \mathrm{~mm}, 50 \mathrm{~mm}, 52 \mathrm{~mm}$, and 40 $\mathrm{mm}$, respectively. Aortic arch vessels and all abdominal branches originated from the true lumen.

Replacement of the thoracic aorta was performed under moderate hypothermic circulatory arrest and ACP. Cardiopulmonary bypass (CPB) was established via a graft to the right axillary artery and right atrium. Cardioplegic arrest was achieved using retrograde cold Custodiol solution. When bladder temperature reached $22.5^{\circ} \mathrm{C}$ and circulatory arrest was established, ACP was started via the right axillary artery and left common carotid artery. The ascending aorta, aortic arch, and proximal descending aorta were resected, and debranching of the aortic arch vessels was performed.

A 24-mm endovascular stent with a 50-mm length graft and 130-mm stent graft (E-vita OPEN PLUS graft, JOTEC, Schweiz, Germany) was implanted in the proximal descending aorta. Arch vessels were selectively implanted on the graft. The proximal ascending aorta and the rest of the aortic arch graft was sutured, and circulation was reestablished. The patient was weaned from

* Corresponding Author:

George Samanidis, MD

Department of Cardiac Surgery

Onassis Cardiac Surgery Center

356 Syggrou Ave., 17674, Athens, Greece

Tel: +003 2109493000; Fax: +003 2109493332; E-Mail: gsamanidis@yahoo.gr 


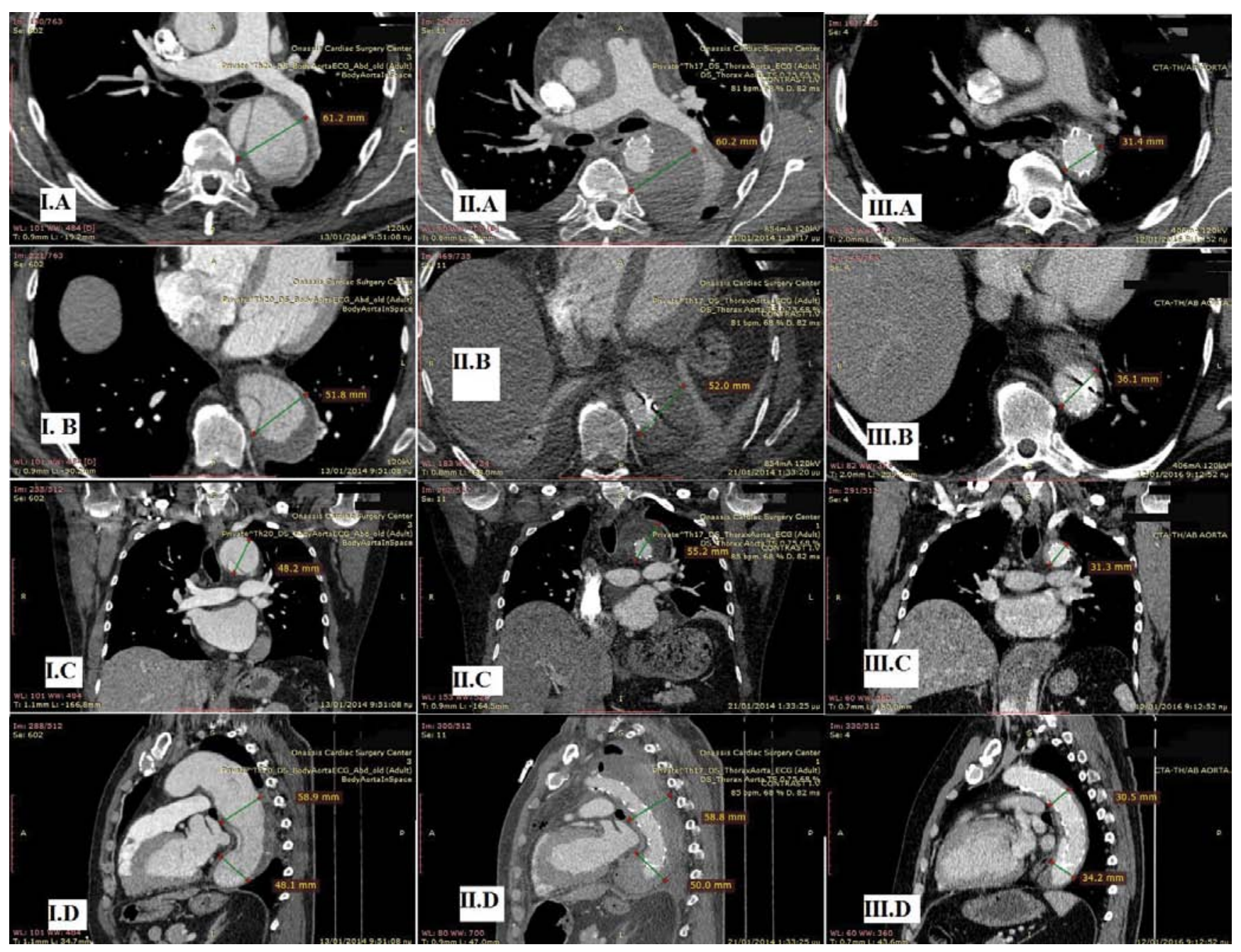

Figure 1. Computed tomography (CT) angiography depicting the evolution of thoracic aorta remodeling during the follow-up period at four different levels (Panel A. axial cut pulmonary level, Panel B: axial cut distal graft level, Panel C. coronal view distal arch level, Panel D. sagittal view pulmonary level and distal graft level). Panel I. Preoperatively. Panel II. Four days after the operation. Panel III. Two years after the operation.
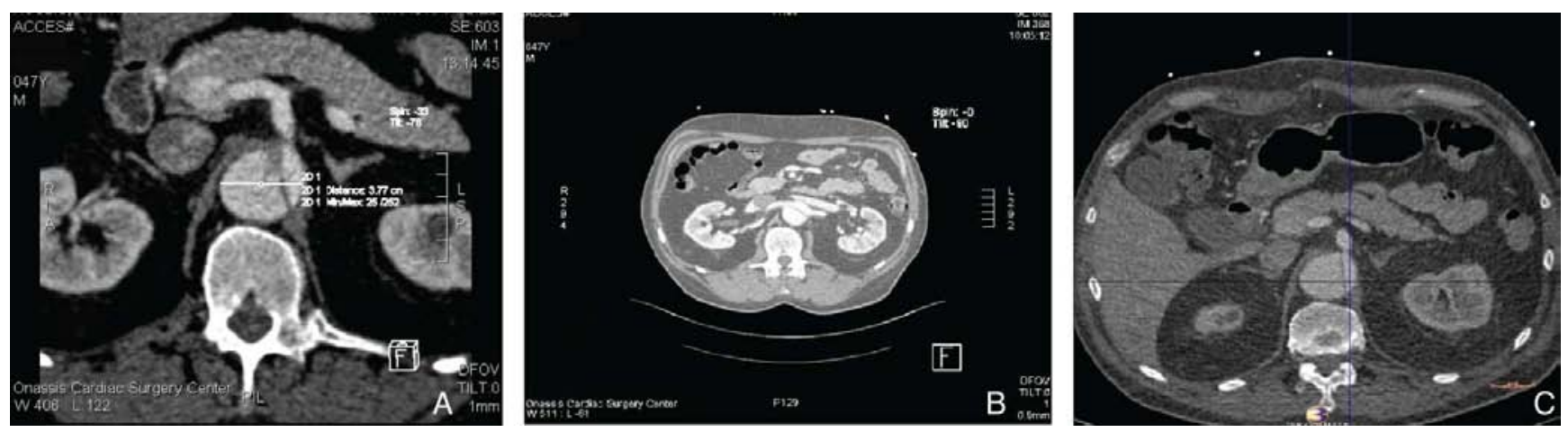

Figure 2. CT angiography showing evolution during the follow-up period. Panel A. Preoperatively. Panel B. Four days after the operation. Panel C. Two years after the operation. 
CPB and transferred to the intensive care unit. Circulatory arrest, aortic cross clamp, and CPB durations were $93 \mathrm{~min}, 146 \mathrm{~min}$, and $292 \mathrm{~min}$, respectively. Four days after the operation, CT angiography revealed thrombosis of the false lumen in the descending thoracic aorta (Figures 1IIA, 1IIB, 1IIC, and 1IID). The patient's postoperative course was uneventful, and he was discharged home on postoperative day 9. At 24-month follow-up, CT angiography of the aorta showed complete obliteration and shrinkage of the false lumen of the descending thoracic aorta (Figures $1 \mathrm{IIIA}, 1 \mathrm{III}, 1 \mathrm{IIIC}$, and 1 IIID) and thrombosis of the false lumen of the $a b$ dominal aorta (Figures $2 \mathrm{~B}$ and $2 \mathrm{C}$ ).

The descending thoracic aorta is amenable to remodeling by extending the stent graft into the true lumen with subsequent obliteration of the false lumen. The latter procedure is achieved at all levels of the stent graft. A similar procedure has been considered successful when complete thrombosis of the false lumen is achieved at 3-month follow-up, both in cases of acute and chronic Stanford Type A aortic dissection [1-3]. Gorlitzer et al. [1] described shrinkage of the false lumen in $64 \%$ of patients who underwent this treatment modality across a follow-up period of 12 months. In our case, complete shrinkage of the false lumen was present at 24-month follow-up.

\section{Conflict of Interest}

The authors have no conflict of interest relevant to this publication.

\section{Comment on this Article or Ask a Question}

\section{References}

1. Gorlitzer M, Weiss G, Meinhart J, Waldenberger F, Thalmann M, Folkmann, et al. Fate of the false lumen after combined surgical endovascular repair treating Stanford Type A aortic dissections. Ann Thorac Surg. 2010;89:794799. DOI: 10.1016/j.athoracsur.2009.11.054

2. Di Bartolomeo R, Di Marco L, Armaro A, Marsilli D, Leone A, Pilato E, et al. Treatment of complex disease of the thoracic aorta: the frozen elephant trunk technique with the E-vita open prosthesis. Eur J Cardiothorac Surg. 2009;35:671-676. DOI: 10.1016/j. ejcts.2008.12.010

3. Huptas S, Mehta RH, Kühl H, Tsagakis K, Reinsch N, Kahlert $\mathrm{P}$, et al. Aortic remodeling in Type $B$ aortic dissection: effects of endovascular stent-graft repair and medical treatment on true and false lumen volumes. J Endovasc Ther. 2009;16:28-38. DOI: 10.1583/08-2497.1
Cite this article as: Samanidis $G$, Kanakis $M$, leromonachos C, Stavridis G. Complete Shrinkage of the Obliterated False Lumen After Open and Endovascular Chronic Aortic Dissection Stanford Type A Repair. AORTA (Stamford). 2017;5(2):61-63. DOI: https://doi.org/10.12945/j. aorta.2017.16.035 